\title{
A TOPOLOGICAL CLASSIFICATION OF DIFFERENTIAL EQUATIONS WITH IMPULSE EFFECT
}

\author{
D. D. BAINOV ${ }^{1}$, S. I. KOSTADINOV ${ }^{1}$, NGUYEN VAN MINH ${ }^{2}$ AND P. P. ZABREIKO ${ }^{2}$
}

\begin{abstract}
A.bstract. The present paper is concerned with the problem of topological classification of differential equations with impulse effect. Under the assumption that the linear part of the right-hand side of the equation considered has an exponential dichotomy and the nonlinear perturbation is small enough it is proved that for the underlying equations there exist $N+1$ types topologically different from one another.
\end{abstract}

\section{Introduction}

Differential equations with impulse effect have been qualitatively investisgated by many authors, see [1], [6], [7] and the references there. A special attention is focussed on the existence of integral manifolds, the dichotomy of solutions (see [1], [7], [10]).

The present paper is concerned with the problem of topological classification of impulsive differential equations. To do this we shall make use of the method developed in [2] for nonautonomous differential equations. It should be noted that the main difficulty we are faced with is due to the discontinuity of the trajectories of impulsive differential equations. To overcome this we shall modify the techniques of constructing a homeomorphism using the Morse lemma (see Lemma 4 and Theorem 1 below). As in [2], introducing the notion of topological equivalence between "proper" integral manifolds we shall prove that the equations

$$
\begin{aligned}
& \frac{d x}{d t}=A(t) x+f(t, x) \quad \text { if } t \neq t_{n} \\
& x\left(t_{n}^{+}\right)=Q_{n} x\left(t_{n}\right)+h\left(x\left(t_{n}\right)\right)
\end{aligned}
$$

Received July 1, 1992.

1980 Mathematics Subject Classification (1985 Revision). Primary 34C35

Key words and phrases. Proper integral manifold, topological equivalence, exponential dichotomy.

${ }^{1}$ Plovdiv University "Paissii Hilendarski", Plovdiv, Bulgaria. Supported by the Bulgarian Ministry of Science and Higher Eduction under Grant MM-7.

${ }^{2}$ Byelorussian State University, Minsk. 
are topologically equivalent to the standard equations

$$
\dot{x}_{1}=-x_{1}, \dot{x}_{2}=x_{2},\left(x_{1}, x_{2}\right)=x
$$

under the assumption that the linear part has an exponential dichotomy and the nonlinear perturbation is small enough.

\section{Statement of the problem}

Suppose $T=\left\{t_{n}: n \in \mathbb{Z}\right\}$ is a sequence of moments in $\mathbb{R}$ satisfying the following conditions:

$$
\begin{aligned}
& \text { i) } t_{n}<t_{n+1} \text { for every } n \in \mathbb{Z}, \lim _{n \rightarrow \pm \infty} t_{n}= \pm \infty \\
& \text { ii) } \lim _{h \rightarrow \infty} \frac{i(t, t+h)}{h}=p<\infty
\end{aligned}
$$

uniformly in $t \in \mathbb{R}$, where $i(a, b)$ denotes the number of moments contained in the interval $(a, b)$.

Consider the impulsive equation

$$
\begin{aligned}
& \frac{d x}{d t}=A(t) x+f(t, x) \quad \text { if } t \neq t_{n} \\
& x\left(t_{n}^{+}\right)=Q_{n} x\left(t_{n}\right)+h_{n}\left(x\left(t_{n}\right)\right)
\end{aligned}
$$

where $t \in \mathbb{R}, x \in \mathbb{R}^{N}, A(\cdot)$ is a matrix-valued function, $Q_{n}$ is a matrix, under the assumption that

i) $A(\cdot)$ and $f(\cdot, \cdot)$ are extendable to continuous ones on evrey set of the form $\left[t_{n}, t_{n+1}\right]$ and $\left[t_{n}, t_{n+1}\right] \times \mathbb{R}^{N}$ respectively.

ii) $Q_{n}$ is invertible for every $n \in \mathbb{Z}$.

iii) $\sup _{\|}\|A(t)\|<\infty, \sup \left\|Q_{n}\right\|<\infty, \sup \|f(t, 0)\|<\infty, \sup \left\|h_{n}(0)\right\|<\infty$.

iv) $\|f(t, x)-f(t, y)\| \stackrel{n}{\leq} \delta\|x-y\|,\left\|h_{n}(x)-h_{n}(y)\right\| \leq \delta\left\|x^{n}-y\right\|$. for all $x, y \in \mathbb{R}^{N}, t \in \mathbb{R}, n \in \mathbb{Z}$.

Definition 1. A solution of the impulsive equation (5), (6) we shall call a function satisfying equation (5) for $t \neq t_{n}$ and equation (6) for $t=t_{n}$ and being continuous from the left.

Remark. Under the above assumption the impulsive equation (5),(6) satisfies all conditions of the Existance and Uniqueness Theorem. So we denote by $U(t, s)$ the Cauchy matrix of the homogeneous equation corresponding to (5), (6) and by $X(t, s, x)$ the solution of (5), (6) satisfying $X(s, s, x)=x$ for every $t \in \mathbb{R}$ and $x \in \mathbb{R}^{N}$. 


\section{Preparatory lemmas}

Before stating the preparatory lemmas we shall use to prove the main result, we shall need the following notions.

Definition2. An intergral manifold of the impulsive equation (5), (6) is said to be proper if $\mathbb{R}^{N}$ splits up into a direct sum of $\mathbb{R}^{k}$ and $\mathbb{R}^{m}$ so that this intergral manifold is represented by the equation

$$
x_{2}=\varphi\left(t, x_{1}\right), x_{1} \in \mathbb{R}_{1}^{k}, x_{2} \in \mathbb{R}^{m}
$$

where $\varphi$ is extendable to a continuous mapping on every set of the from $\left[t_{n}, t_{n+1}\right] \times \mathbb{R}^{k}$, furthermore $\|\varphi(t, x)-\varphi(t, y)\| \leq \eta\|x-y\|$ for all $x, y \in \mathbb{R}^{k}, \eta$ is independent of $t$. $\varphi(t, 0)=0$ for every $t \in \mathbb{R}$.

Definition 3. Let $M, N$ be proper integral manifolds of two given impulsive equations. $M$ is said to be topologically equivalence to $N$ if there exists a homeomorphism $H: M \rightarrow N$ with the properties:

i) $H(t, x)=\left(\theta(t), h_{t}(x)\right)$, where $h_{t}: M(t) \rightarrow N(\theta(t))$ is a homeomorphism, $M=$ $\{(t, M(t)), t \in \mathbb{R}\}, N=\{(t, N(t))\}, \theta: \mathbb{R} \rightarrow \mathbb{R}$ is an orientation-preserving homeomorphism, $\theta\left(t_{n}\right)=\tau_{n}$ for every $n \in \mathbb{Z},\left\{t_{n}: n \in \mathbb{Z}\right\},\left\{\tau_{n}: n \in \mathbb{Z}\right\}$ are moments of impulse effect of the given equations.

ii) If $x(t)$ is any solution contained in $M$, then $h_{t}(x(t))=y(\theta(t))$ where $y(\tau)$ is a solution contained in $N . h_{t}^{-1}$ has the same property.

iii) There exists an increasing function $L:[0, \infty) \rightarrow[0, \infty), L(0)=0$, continuous at 0 and such that

$$
\begin{aligned}
& \left\|h_{t}(x)\right\| \leq L(\|x\|) \quad \text { for every }(t, x) \in M \\
& \left\|h_{t}^{-1}(y)\right\| \leq L(\|y\|) \quad \text { for every }(t, y) \in N .
\end{aligned}
$$

Remark $\mathbb{1}$. i) The above defined topological equivalence is an equivalence relation.

ii) If $\left\{t_{n}: n \in \mathbb{Z}\right\},\left\{\tau_{n}: n \in \mathbb{Z}\right\}$ satisfy condition (3), (4) then the topological equivalence preserves the boundedness of solutions and the stability of the trivial solution.

iii) From the condition iii) imposed on $h_{t}, h_{t}^{-1}$ we deduce that

$$
\lim _{\|x\| \rightarrow \infty}\left\|h_{t}(x)\right\|=\lim _{\|y\| \rightarrow \infty}\left\|h_{t}^{-1}(y)\right\|=\infty
$$

uniformly.

From now on we shall deal only with impulsive equations with moments of impulse effect satisfying (3), (4), and integral manifolds satisfying the conditions in Definition 2 except for the condition $\varphi(t, 0)=0$ for all $t \in \mathbb{R}$.

Definition 4. Let $M, N$ be integral manifolds of two given impulsive equations. $M$ is said to be topologically weakly equivalent to $N$ if there exists a homeomorphism $H: M \rightarrow N$ satisfying conditions i), ii) in Definition 3 and equality (8). 
So if $M, N$ are proper and topologically equivalent, then they are topologically weakly equivalent to each other. It may be noted that weak topological equivalence is an equivalence relation. This preserves the boundedness of solutions.

Definition 5. The homogeneous equation corresponding to (1), (2) is said to have an exponential dichotomy if there exist positive constants $M, \alpha$ and a projector $P: \mathbb{R}^{N} \rightarrow \mathbb{R}^{N}$ such that

$$
\begin{aligned}
& \left\|X(t) P X^{-1}(s)\right\| \leq M \exp (-\alpha(t-s)) \quad \text { for } t \geq s \\
& \left\|X(t)(I-P) X^{-1}(s)\right\| \leq M \exp (-\alpha(s-t)) \quad \text { for } t \leq s
\end{aligned}
$$

where $X(t)$ is a fundamential matrix of the homogeneous equation.

Now consider two linear systems

and

$$
\begin{aligned}
& \dot{x}=A(t) x \quad \text { if } t \neq t_{n} \\
& x\left(t_{n}^{+}\right)=Q_{n} x\left(t_{n}\right)
\end{aligned}
$$

$$
\begin{aligned}
& \dot{x}=B(t) x \quad \text { if } t \neq t_{n} \\
& x\left(t_{n}^{+}\right)=R_{n} x\left(t_{n}\right)
\end{aligned}
$$

Definition 6. Equation (11), (12) is said to be kinematically similar to equation $(9),(10)$ if there exists a matrix-valued function $S(\cdot)$ having the following properties:

i) $S(\cdot)$ is continuous for $t \neq t_{n}$ and bounded on $\mathbb{R}$.

ii) $S(t)$ has discontinuities of the first kind at $t=t_{n}$ and is continuous from the left.

iii) $S(t)$ is invertible for every $t \in \mathbb{R}$ and $S^{-1}(\cdot)$ enjoys the properties i), ii).

iv) If $x(t)$ is any solution of (11), (12), then $S(t) x(t)$ is a solution of (9), (10).

Lemma 1. Assume that equation (9), (10) has an exponential dichotomy. Then it is kinematically similar to a reducible equation (11), (12), i.e $B(t), \mathbb{R}_{n}$ commute with the projector $P$, in addition, $\|B(t)\| \leq\|A(t)\|,\left\|R_{n}\right\| \leq\left\|Q_{n}\right\|$ for all $t \in \mathbb{R}, n \in \mathbb{Z}$.

This lemma is proved by modifying the proof of the well-know result on reducibility (see [4], [5], [9], [10]).

Lemma 2. Suppose that the homogeneous equation corresponding to (5), (6) has an exponential dichotomy with projector $P$ and positive constants $M$, $\alpha$, and enjoys the property $f(t, 0)=0, h_{n}(0)=0$. Then for sufficiently small $\delta$ there exist proper integral manifolds

$$
\begin{array}{ll}
M^{-}=\left\{(s, x) \in \mathbb{R} \times \mathbb{R}^{N}:\|X(t, s, x)\| \rightarrow 0\right. & \text { as } t \rightarrow \infty\} \\
M^{+}=\left\{(s, x) \in \mathbb{R} \times \mathbb{R}^{N}:\|X(t, s, x)\| \rightarrow 0\right. & \text { as } t \rightarrow-\infty\} .
\end{array}
$$


Furthermore, if $f(t, \cdot), h(\cdot)$ are of class $C^{k}$, where $k \in \mathbb{N}$ so are these intergral manifolds.

For the proof see [6]. It may be noted that if $f(t, \cdot),{ }^{i} h_{n}(\cdot)$ are of class $C^{k}$, we can show that these integral manifolds are of class $C^{k}$, too.

Lemma 3. Suppose all conditions in Lemma 2 are satisfied except for $f(t, 0)=0, h_{n}(0)=0$. Then equation (1), (2) has at least one bounded solution.

For the it proof see [6], too.

\section{Main results}

Lemma 4. Let the homogeneous equation corresponding to (5), (6) have an exponential dichotomy with projector $P=O$ and positive constants $M, \alpha$, in addition, $f(t, 0)=0, h_{n}(0)=0, f(t, \cdot), h_{n}(\cdot) \in C^{3}$. Then for $\delta$ small enough equation (5), (6) is topologically equivalent to the standard equation

$$
\dot{x}=x, \quad x \in \mathbb{R}^{N}
$$

Proof. The main difficulty in proving this lemma is due to the discontinuity of the trajectories of solutions of equation (5), (6). But the main idea of the proof is suggested by [3]. First we consider the function

$$
V(t, x)=\int_{-\infty}^{t}\|X(\tau, t, x)\|^{2} d \tau
$$

This function is well-defined. In fact, we have the variation of parameters formula

$$
X(t, s, x)=U(t, s) x+\int_{s}^{t} U(t, \tau) f(\tau, X(\tau, s, x)) d \tau+\sum_{s<t_{i} \leq t} U\left(t, t_{i}\right) Q_{i}\left(X\left(t_{i}, s, x\right)\right) .
$$

Setting $\psi(t)=\|X(t, s, x)\|$ we have for $t \leq s$

$$
\begin{gathered}
\|\psi(t)\| \leq M\|x\| \exp (-\alpha(s-t))+\int_{t}^{s} M \exp (-\alpha(\tau-t)) \delta\|\psi(\tau)\| d \tau \\
+\sum_{t<t_{i} \leq s} M \exp \left(-\alpha\left(t_{i}-t\right)\right) \delta\left\|\psi\left(t_{i}\right)\right\|
\end{gathered}
$$

Thus

$$
\begin{gathered}
e^{-\alpha t}\|\psi(t)\| \leq M\|x\| \exp (-\alpha s)+\int_{t}^{s} \delta M \exp (-\alpha \tau)\|\psi(\tau)\| d \tau \\
+\sum_{t<t_{i} \leq s} \delta M \exp \left(-\alpha t_{i}\right)\left\|\psi\left(t_{i}\right)\right\| .
\end{gathered}
$$


Putting $u(t)=e^{-\alpha t}\|\psi(t)\|$ and applying Gronwall's inequality (see [6], [7]), we get

$$
u(t) \leq \sqcap_{t<t_{i}<s}(1+M \delta) M\|x\| \exp (-\alpha s) \exp (M \delta(s-t))
$$

So we have

$$
\|\psi(t)\| \leq(1+M \delta)^{i(t, s)} M\|x\| \exp (-(\alpha-M \delta)(s-t))
$$

From this it follows that for $\delta$ small enough the integral (13) is absolutely convergent, uniformly with respect to $x$ contained in an arbitrary bounded set of $\mathbb{R}^{N}$.

From now on we assume that $\delta$ is chosen so small that

$$
(1+M \delta)^{i(t, s)} M\|x\| \exp (-(\alpha-M \delta)(s-t)) \leq M\|x\| \exp (-\bar{\alpha}(s-t)),
$$

where $\bar{\alpha}$ is a fixed positive constant.

Note that $V(\cdot, x)$ is continuous at $t \neq t_{n}$ and has a discontinuity of the first kind at $t=t_{n}$. To prove Lemma 4 we need the following

Lemma 5. For $\delta$, $\varepsilon$ small enough, $s$ fixed, the set $\left\{x \in \mathbb{R}^{N}: V(s, x)=\varepsilon\right\}$ is homeomorphic to $\left\{x \in \mathbb{R}^{N}:\|x\|=1\right\}$, in addition, if we denote by $g_{s}$ that homeomorphism, then $g_{s}(x), g_{s}^{-1}(x)$ depend on $(s, x)$ continuously for $s \neq t_{n}$ and have a discontinuity of the first kind at $s=t_{n}$. have

Proof. We shall make use of the Morse lemma. By calculating $\left.D_{x}^{2} V(s, x)\right|_{x=0}$ we

$$
\begin{aligned}
\left.D_{x} V(s, x)\right|_{x=0}(\xi)= & \int_{-\infty}^{s} 2\left\langle D_{x} X(u, s, 0) \xi, X(u, s, 0)\right\rangle d u=0 \\
\left.D_{x}^{2} V(s, x)\right|_{x=0}(\xi, \eta)= & 2 \int_{-\infty}^{s}\left\langle D_{x}^{2} X(u, s, 0)(\xi, \eta), X(u, s, 0)\right\rangle d u \\
& +2 \int_{-\infty}^{s}\left\langle D_{x} X(u, s, 0) \xi, D_{x} X(u, s, o) \eta\right\rangle d u \\
= & 2 \int_{-\infty}^{s}\left\langle D_{x} X(u, s, 0) \xi, D_{x} X(u, s, 0) \eta\right\rangle d u
\end{aligned}
$$

We are going to show that

$$
\int_{-\infty}^{s}\left\|D_{x} X(u, s, 0) \xi\right\|^{2} d u \geq c\|\xi\|^{2}
$$

for some positive $C$. In fact, from (14) we deduce that

$$
\begin{aligned}
D_{x} X(u, s, 0)= & U(u, s)+\int_{u}^{s} U(u, \tau) D_{2} f(\tau, X(\tau, s, 0)) D_{x} X(\tau, s, 0) d \tau \\
& +\sum_{u<t_{i} \leq s} U\left(u, t_{i}\right) D Q_{i}\left(X\left(t_{i}, s, 0\right)\right) D_{x} X(u, s, 0)
\end{aligned}
$$


Without difficulty we may show that

$$
\left\|D_{x} X(u, s, 0)\right\| \leq M \exp (-\bar{\alpha}(s-u)) \quad \text { for } u \leq s
$$

Hence

$$
\begin{aligned}
& \int_{-\infty}^{s}\left\|D_{x} X(u, s, 0) \xi\right\|^{2} d u \\
= & \int_{-\infty}^{s}\|U(u, s) \xi\|^{2} d u+\int_{-\infty}^{s} \|\left(\int_{u}^{s} U(u, \tau) D_{2} f(\tau, 0) D_{x} X(\tau, s, 0) d \tau\right. \\
& \left.+\sum_{u<i_{i} \leq s}^{s} U\left(u, t_{i}\right) D Q_{i}(0) D_{x} X(u, s, 0)\right)(\xi) \|^{2} d u \\
& +2 \int_{-\infty}^{s}\left\langle U(u, s) \xi,\left(\int_{u}^{s} U(u, \tau) D_{2} f(\tau, 0) D_{x} X(\tau, s, 0) d \tau\right.\right. \\
& \left.\left.+\sum_{u<t_{i} \leq s} U\left(u, t_{i}\right) D Q_{i}(0) D_{x} X(u, s, 0)\right)(\xi)\right\rangle d u
\end{aligned}
$$

Taking into account (18), we can show without difficulty that

$$
\int_{-\infty}^{s}\left\|D_{x} X(u, s, 0) \xi\right\|^{2} d u \geq \int_{-\infty}^{s}\|U(u, s) \xi\|^{2} d u-\delta \bar{M}\|\xi\|^{2}
$$

where $\bar{M}$ is a positive constant independent of $\xi$.

Since the linear part of (1) has an exponential dichotomy with projector $P=0$, then (see[4]) there exists a constant $C_{1}>0$ such that

$$
\int_{-\infty}^{s}\|U(u, s) \xi\|^{2} d u \geq c_{1}\|\xi\|^{2}
$$

Finally, if $\delta$ is a chosen small enough, we get

$$
\left.D_{x}^{2} V(s, x)\right|_{x=0}(\xi, \xi) \geq c\|\xi\|^{2}
$$

where $c$ is a positive constant.

Now, making use of the Morse lemma, we see that for $\varepsilon$ small enough the set $\{x \in$ $\left.\mathbb{R}^{N}: V(s, x)=\varepsilon\right\}$ is homeomorphic to the unit sphere (see [8] for the details). In addition, from the proof of the Morse lemma we deduce that $g_{s}(x)$ depends on $(s, x)$ continuously for $s \neq t_{n}$ and has a discontinuity at $s=t_{n}$. This completes the proof of Lemma 5.

Now we continue with the proof of Lemma 4 . Under the smallness assumption of $\delta$ we have (16) and then for $t>\tau$

$$
\|x\|=\|X(\tau, t, X(t, \tau, x))\| \leq M\|X(t, \tau, x)\| \exp (-\bar{\alpha}(t-\tau))
$$


Thus

$$
M^{-1}\|x\| \exp (\bar{\alpha}(t-\tau)) \leq\|X(t, \tau, x)\| \quad \text { for } t>\tau
$$

Let $x(t)$ denote $X(t, 0, x)$. It is easily shown that $V(t, x(t))$ is continuous on the whole axis. Furthermore, at $t \neq t_{n}$

$$
\begin{aligned}
\frac{d}{d t} V(t, x(t)) & =\frac{d}{d t} \int_{-\infty}^{t}\|X(\tau, 0, x)\|^{2} d x \\
& =\|X(t, 0, x)\|^{2}=\|x(t)\|^{2}
\end{aligned}
$$

At $t \neq t_{n}$ we have

$$
\begin{aligned}
D_{-} V\left(t_{n}, x\left(t_{n}\right)\right) & =\lim _{k \rightarrow 0} \frac{1}{k}\left[V\left(t_{n}, x\left(t_{n}\right)\right)-V\left(t_{n}-k, x\left(t_{n}-k\right)\right)\right] \\
& =\left\|X\left(t_{n}, 0, x\right)\right\|^{2}=\left\|x\left(t_{n}\right)\right\|^{2}
\end{aligned}
$$

From (16) it follows that

$$
V(t, x) \leq \int_{-\infty}^{t} M^{2}\|x\|^{2} \exp (-2 \bar{\alpha}(t-\tau)) d \tau \leq \frac{M^{2}}{2 \bar{\alpha}}\|x\|^{2}
$$

Set $K=\sup _{t}\|A(t)\|+\delta$. For $\tau, t \in\left(t_{n}, t_{n+1}\right)$ we have no difficulty in proving that

$$
\frac{d}{d t} \ln \|X(t, \tau, x)\| \leq K
$$

Thus

$$
\|X(t, \tau, x)\| \leq\|x\| \exp (K(t-\tau)) \quad \text { for } t>\tau
$$

Now for $t_{k}<\tau \leq t_{k+1}<\ldots<t_{n}<t \leq t_{n+1}$ we have

$$
\|X(t, \tau, x)\| \leq L^{i(\tau, t)}\|x\| \exp (K(t-\tau))
$$

where $L=\sup _{n}\left\|Q_{n}\right\|+\delta$. So we can find a positive number $K_{1}$ such that

$$
\|X(t, \tau, x)\| \leq\|x\| \exp \left(K_{1}(t-\tau)\right) \quad \text { for } t>\tau
$$

For the case when $t<\tau$ we have

$$
\|X(\tau, t, X(t, \tau, x))\| \leq\|X(t, \tau, x)\| \exp \left(K_{1}(\tau-t)\right)
$$

Hence for $t<\tau$

$$
\|x\| \exp \left(K_{1}(t-\tau)\right) \leq\|X(t, \tau, x)\|
$$


Taking into account (23), we have

$$
\begin{aligned}
V(t, x) & =\int_{-\infty}^{t}\|X(s, t, x)\|^{2} d s \geq \int_{-\infty}^{t}\|x\|^{2} \exp \left(2 K_{1}(s-t)\right) d s \\
& \geq \frac{1}{2 K_{1}}\|x\|^{2}
\end{aligned}
$$

Consider the function $f(t, s, x)=V(t, X(t, s, x))$. For $t \neq t_{n}$

$$
\frac{\partial f}{\partial t}(t, s, x)=\frac{d}{d t} V(t, X(t, s, x))=\|X(t, s, x)\|^{2}
$$

Combining (21) and $(24)$, we have

$$
\frac{M^{2}}{2 \bar{\alpha}}\|X(t, s, x)\|^{2} \geq f(t, s, x) \geq \frac{1}{2 K_{1}}\|X(t, s, x)\|^{2}
$$

From (16), (19) it follows that for $x \neq 0$

$$
\lim _{t \rightarrow-\infty}\|X(t, s, x)\|=0, \quad \lim _{t \rightarrow \infty}\|X(t, s, x)\|=\infty
$$

Hence

$$
\lim _{t \rightarrow-\infty} f(t, s, x)=0, \quad \lim _{t \rightarrow \infty} f(t, s, x)=\infty
$$

It is easily shown that the equation $\varepsilon=f(t, s, x)$ has a unique solution $t=t(s, x)$, where $\varepsilon$ is chosen as in Lemma 5 , furthermore $t$ depends on $(s, x)$ continuously when $x \neq 0$.

Now we are in a position to construct the homeomorphism which transforms the underlying equation into the standard one. In fact, we define the homeomorphism $H$ : $\mathbb{R} \times \mathbb{R}^{N} \rightarrow \mathbb{R} \times \mathbb{R}^{N}$ as follows

$$
\left\{\begin{array}{l}
\left.h_{s}(x)=\exp (s-t(s, x)) g_{t(s, x)}(X(t, s, x), s, x)\right) \text { for } x \neq 0 \\
h_{s}(0)=0
\end{array}\right.
$$

where $g_{s}$ is defined in Lemma 4. We are going to show that $H$ satisfies all properties listed in Definition 3. In fact, for $x \neq 0, h_{s}(x)$ is continuous with respect to $(s, x)$. For $x=0$

$$
\left\|h_{s}(x)-h_{s}(0)\right\| \leq \exp (s-t(s, x))
$$

We shall estimate the right-hand side of (26). By definition we have

$$
\begin{aligned}
V(s, x)-\varepsilon & =V(s, x)-V(t(s, x), X(t, s, x), s, x)) \\
& =\int_{t(s, x)}^{s} D_{-} V(u, X(u, s, x)) d u
\end{aligned}
$$

Hence

$$
|s-t(s, x)| \leq|V(s, x)-\varepsilon| / \inf _{u \in[t(s, x), s], u \neq t_{n}} \frac{\partial f}{\partial u}(u, s, x)
$$


If $V(s, x) \geq \varepsilon$, we have $t(s, x) \leq s$. For $u \in[t(s, x), s]$ we obtain

$$
\varepsilon \leq f(u, s, x) \leq \frac{M^{2}}{2 \bar{\alpha}}\|X(u, s, x)\|^{2}
$$

Thus

$$
\frac{\partial f}{\partial u}(u, s, x)=\|X(u, s, x)\|^{2} \geq 2 \varepsilon \bar{\alpha} / M^{2},
$$

for $u \in[t(s, x), s], u \neq t_{n}$. So we get

$$
0 \leq s-t(s, x) \leq(V(s, x)-\varepsilon) \cdot \frac{M^{2}}{2 \varepsilon \bar{\alpha}} \leq\left(\frac{M^{2}}{2 \bar{\alpha}}\|x\|^{2}-1\right) \frac{M^{2}}{2 \varepsilon \bar{\alpha}}
$$

Taking into account (24), we see that we have (29) when

$$
\|x\| \geq \sqrt{\varepsilon / 2 K_{1}}
$$

For $\|x\| \leq \sqrt{2 \varepsilon \bar{\alpha}} / M$ we have $\varepsilon \geq V(s, x)$. So from (22), (27) we get

$$
\begin{aligned}
0 \leq \varepsilon-V(s, x) & \leq\|x\|^{2} \int_{s}^{t(s, x)} \exp \left(2 K_{1}(u-s)\right) d u \\
& \leq \frac{\|x\|^{2}}{2 K_{1}}\left(\exp \left(2 K_{1}(t(s, x)-s)\right)-1\right)
\end{aligned}
$$

Thus

$$
\begin{aligned}
& 0 \leq \varepsilon-\frac{M^{2}}{2 \bar{\alpha}}\|x\|^{2} \leq \frac{\|x\|^{2}}{2 K_{1}}\left(\exp \left(2 K_{1}(t(s, x)-s)\right)-1\right) \\
& \frac{1}{2 K_{1}} \ln \left[\left(\varepsilon-\left(\frac{M^{2}}{2 \bar{\alpha}}+\frac{1}{2 K_{1}}\right)\|x\|^{2}\right) \frac{2 K_{1}}{\|x\|^{2}}\right] \leq t(s, x)-s
\end{aligned}
$$

From this it follows that $h_{s}(x)$ is continuous at $x=0$. Furthermore we easily see that there exists an increasing function $L:[0, \infty) \rightarrow[0, \infty), L(0)=0$, continous at 0 such that

$$
\left\|h_{s}(x)\right\| \leq L(\|x\|) \quad \text { for } x \in \mathbb{R}^{N}
$$

It is easily checked that $H^{-1}: \mathbb{R} \times \mathbb{R}^{N} \rightarrow \mathbb{R} \times \mathbb{R}^{N}$ is defined by the following formula

$$
\begin{aligned}
& H^{-1}(s, x)=\left(s, h_{s}^{-1}(x)\right), \quad \text { where } \\
& h_{s}^{-1}(x)=X\left(s, u, g_{u}^{-1}(x /\|x\|), u=s-\ln \|x\| \quad \text { for } x \neq 0\right. \\
& h_{s}^{-1}(0)=0
\end{aligned}
$$

It is obvious that for $x \neq 0, h_{s}^{-1}(x)$ is continuous with respect to $(s, x)$. For $\|x\|<1$ we have $u>s$. So from (16), (24) it follows that

$$
\begin{aligned}
\left\|h_{s}^{-1}(x)\right\| & \leq M\left\|g_{u}^{-1}(x /\|x\|)\right\| \exp (-\bar{\alpha}(u-s)) \\
& \leq M \frac{\sqrt{\varepsilon}}{2 K_{1}} \exp (\bar{\alpha} \ln \|x\|)=M \frac{\sqrt{\varepsilon}}{2 K_{1}}\|x\|^{\bar{\alpha}}
\end{aligned}
$$


For $\|x\|>1$ we have

$$
\left\|h_{s}^{-1}(x)\right\| \leq\left\|g_{u}^{-1}(x /\|x\|) \exp \left(K_{1}(s-u)\right) \leq \frac{\sqrt{\varepsilon}}{2 K_{1}}\right\| x \|^{K_{1}}
$$

From (32), (33) it is seen that $h_{s}^{-1}$ is continuous at 0 , and there exists an increasing function $L^{\prime}:[0, \infty) \rightarrow[0, \infty), L^{\prime}(0)=0$, continuous at 0 such that

$$
\left\|h_{s}^{-1}(x)\right\| \leq L^{\prime}(\|x\|) \quad \text { for every }(s, x) \in \mathbb{R} \times \mathbb{R}^{N}
$$

This completes the proof of Lemma 4.

Theorem 1. Let the homogeneous equation correspomding to (5), (6) have an exponential dichotomy with projector $P$ and positiove constants $M, \alpha, f(t, 0)=$ $h_{n}(0)=0$ for all $t, n$, let $f(t, \cdot), h_{n}(\cdot)$ be of class $C^{3}$. Then for $\delta$ small enough equation (5), (6) is topologically equivalent to the standard equation

$$
\begin{cases}\dot{x}_{1}=-x_{1}, & x_{1} \in \mathbb{R}^{k} \\ \dot{x}_{2}=x_{2}, & x_{2} \in \mathbb{R}^{m}\end{cases}
$$

where $k=\operatorname{dim} P\left(\mathbb{R}^{N}\right)$.

Proof. We shall prove this theorem applying the idea of the proof of Theorem in [2]. Frist, $Q_{n}, A(t)$ can be assumed to commute with $P$. Under the assumtions of the theorem for every $(s, x) \in \mathbb{R} \times \mathbb{R}^{N}$ there exist

$$
\begin{aligned}
& E^{-}(s, x)=\left\{y \in \mathbb{R}^{N}:\|X(t, s, y)-X(t, s, x)\| \rightarrow 0, \text { as } t \rightarrow \infty\right\} \\
& E^{+}(s, x)=\left\{y \in \mathbb{R}^{N}:\|X(t, s, y)-X(t, s, x)\| \rightarrow 0, \text { as } t \rightarrow-\infty\right\}
\end{aligned}
$$

Suppose $\varphi_{s}^{-}: E^{k}=P\left(\mathbb{R}^{N}\right) \rightarrow E^{m}=(I-P)\left(\mathbb{R}^{N}\right), \varphi_{s}^{+}: E^{m} \rightarrow E^{k}$ such that

$$
\begin{aligned}
& E^{-}(s, 0)=\left\{x+\varphi_{s}^{-}(x), x \in E^{k}\right\} \\
& E^{+}(s, 0)=\left\{\varphi_{s}^{+}(x)+x, x \in E^{m}\right\}
\end{aligned}
$$

Here, for the sake of convenience, we assume that the scalar product is chosen so that $P$ is an orthogonal projection. We denote

$$
E^{-}=\left\{\left(s, E^{-}(s, 0)\right), s \in \mathbb{R}\right\}, \quad E^{+}=\left\{\left(s, E^{+}(s, 0)\right), s \in \mathbb{R}\right\} .
$$

Then both $E^{-}, E^{+}$are proper integral manifolds. We shall establish the topological equivalence between $E^{-}, E^{+}$and $E^{k}, E^{m}$. Suppose $x(t)$ is any solution of (5), (6) contained in $E^{-}$. Then $P_{x}(t)$ satisfies the following equation

$$
\begin{aligned}
\frac{d}{d t} P_{x}(t) & =P \frac{d x(t)}{d t}=P^{2} A(t) x(t)+P f(t, x(t)) \\
& =P^{2} A(t) x(t)+P f(t, x(t)) \\
& =(P A(t)) P x(t)+P f(t, x(t)) \quad \text { if } t \neq t_{n} \\
P_{x}\left(t_{n}^{+}\right) & =P Q_{n} x\left(t_{n}\right)+P h_{n}\left(x\left(t_{n}\right)\right) \\
& =P^{2} Q_{n} x\left(t_{n}\right)+P h_{n}\left(x\left(t_{n}\right)\right) \\
& =\left(P Q_{n}\right) P x\left(t_{n}\right)+P h_{n}\left(x\left(t_{n}\right)\right)
\end{aligned}
$$


From Lemma 4 it follows that equation (34), (35), setting $u=P x$, is topologically equivalent to $\dot{x}_{1}=-x_{1}, x_{1} \in E^{k}$. Meanwhile, we can easily see that $I \times P$ is a homeomorphism by which $E^{-}$is topologically equivalent to equation (34), (35). Finally we have shown that $E^{-}$is topologically equivalent to the standard equation $\dot{x}_{1}=-x_{1}, x_{1} \in E^{k}$. Similarly, it is shown that $\mathbb{E}^{+}$is topologically equivalent to $\dot{x}_{2}=x_{2}, x_{2} \in \mathbb{E}^{m}$. We denote by $H^{-}(t, x)=\left(t, h_{t}^{-}(x)\right), H^{+}(t, x)=\left(t, h_{t}^{+}(x)\right)$ the homeomorphism which transform the standard equations $\dot{x}_{1}=-x_{1}, \dot{x}_{2}=x_{2}$ into $E^{-}, E^{+}$respecticely. Suppose that $x \in \mathbb{R}^{N}$, $x=u+v, u \in E^{k}, v \in E^{m}$. Setting $a=h_{t}^{-}(u), b=h_{t}^{+}(v)$, we define $H$ as follows

$$
H(t, x)=\left(t, h_{t}(x)\right)
$$

where

$$
h_{t}(x)=E^{+}(t, a) \cap E^{-}(t, b)
$$

It is shown that the definition of $h_{t}$ is correct (see [12]). It is easy to see that $x=$ $h_{t}^{-1}(y)=\left(h_{t}^{-}\right)^{-1}(a)+\left(h_{t}^{+}\right)^{-1}(b)$, where $a=E^{+}(t, y) \cap E^{-}(t, 0), b=E^{-}(t, y) \cap E^{+}(t, 0)$. From the results in [11], [12] it follows that $h_{t}, h_{t}^{-1}$ are both continuous. Now suppose that $x(t)$ is a solution of standard system $\dot{u}=-u, \dot{v}=v, u \in E^{k}, v \in E^{n-k}, x=u+v$. Then $h_{t}(x(t))$ is a solution of (5), (6). In fact, denoting by $Y(t, s, y)$ the solution of the equation

$$
\begin{aligned}
& \frac{d y}{d t}=A(t) y+f(t, y) \quad \text { if } t \neq t_{n} \\
& y\left(t_{n}^{+}\right)=Q_{n} y\left(t_{n}\right)+h_{n}\left(y\left(t_{n}\right)\right) \\
& y(s)=y,
\end{aligned}
$$

we have $Y\left(t, s, E^{-}(s, y)\right)=E^{-}(t, Y(t, s, y)), Y\left(t, s, E^{+}(s, y)\right)=E^{+}(t, Y(t, s, y))$. Suppose $x(t)=u(t)+v(t)$. We put $a(t)=h_{t}^{-}(u(t)), b(t)=h_{t}^{+}(v(t))$. So $(t, a(t)),(t, b(t))$ belong to $E^{-}, E^{+}$, respectively. We get $Y(t, 0, a(0))=a(t), Y(t, 0, b(0))=b(t), Y(t, 0$, $\left.E^{+}(0, a(0))\right)=E^{+}(t, a(t)), Y\left(t, 0, E^{-}(0, b(0))\right)=E^{-}(t, b(t))$. By definition $y(t)=$ $h_{t}(x(t))=E^{+}(t, a(t)) \cap E^{-}(t, b(t))=Y\left(t, 0, E^{+}(0, a(0)) \cap E^{-}(0, b(0))\right)=Y\left(t, 0, h_{0}(x(0))\right)$, i.e. $y(t)$ is a solution of (5), (6). It is easy to see that $h_{t}^{-1}$ has a similar property.

Now we have to prove the existence of a function $L$ with the desired properties. If we choose $\delta$ small enough, we get (see [11], [12])

$$
\left\|y_{2}-b_{2}\right\| \leq \frac{1}{2}\left\|y_{1}-b_{1}\right\|, \quad\left\|y_{1}-a_{1}\right\| \leq \frac{1}{2}\left\|y_{2}-a_{2}\right\|
$$

where $y=\dot{y}_{1}+y_{2}, a=h_{t}^{-}(u)=a_{1}+a_{2}, b=b_{t}^{+}(v)=b_{1}+b_{2} ; y_{1}, a_{1}, b_{1} \in E^{k}$; $y_{2}, a_{2}, b_{2} \in E^{n-k}$. So we get

$$
\left\|y_{1}\right\|+\left\|y_{2}\right\| \leq 2\left(\left\|a_{1}\right\|+\left\|a_{2}\right\|+\left\|b_{1}\right\|+\left\|b_{2}\right\|\right)
$$

Finally, this implies the existence of a funtion $L_{1}:[0, \infty) \rightarrow[0, \infty)$ with the desired properties such that $\left\|h_{t}^{-1}(x)\right\| \leq L_{1}(\|x\|)$. In the same way, there exist $L_{2}:[0, \infty) \rightarrow$ $[0, \infty)$. Finally we choose $L(\|x\|)=\max \left(L_{1}(\|x\|), L_{2}(\|x\|)\right.$ and then we get

$$
\sup _{t \in \mathbb{R}} \max \left(\left\|h_{t}(x)\right\|,\left\|h_{t}^{-1}(x)\right\|\right) \leq L(\|x\|)
$$


This completes the proof of Theorem 1.

Corollary 1. Suppose all conditions of Theorem 1 satisfied except for the condition $f(t, 0)=h_{n}(0)=0$. Then equation (5), (6) is topologically weakly equivalent to the standard equation.

Proof. From Lemma 3 it follows that there exists a bounded solution $\bar{x}(t)$ of equation (5), (6). Consider $h_{t}(x)=\bar{x}(t)+x$. It is easily seen that $H(t, x)=\left(t, h_{t}(x)\right)$ is a homeomorphism which transforms equation (5), (6) into another one satisfying all conditions of Theorem 1. This completes the proof of Corollary 1.

Remark 2. In the present paper the finite dimensionality of the phase space of $X$ is needed only to apply the Morse lemma in Lemma 5. We do not know whether this may be omitted as in case of differential equations without impulse effect.

\section{References}

[1] D. D. Bainov, S. I. Kostadinov and P. P. Zabreiko, "Exponential dichotomy of linear impulsive differential equations in a Banach space", International J. of Theoretical Physics, v. 28(1989), no. 7, 797-814.

[2] Nguyen Van Minh, "On a topological classification of nonautonomous differential equations", Colloquia Mathematica Societaties Janos Bolyai, 53 (1988), 421-426.

[3] J. K. Palmer, "A characterization of exponential dichotomy in terms of topological equivalence", J. Math. Anal. Appl., 69 (1979), No. 1, 8-16.

[4] W. A. Coppel, "Dichotomies in Stability Theory", Lecture Notes in Mathematics, v. 629, SpringerVerlag, Berlin, 1978, p.97.

[5] Ju. L. Daleckii and M.G. Krein, "Stability of Solutions of Differential Equations in Banach Space", AMS, Books and Journals in Advanced Mathematics, 1974, p.386.

[6] A. M. Samoilenko and N. A. Perestyuk, "Differential Equations with Impulse Effecț", Višča S̆kola, Kiev, 1987, p. 287. (in Russian).

[7] D. D. Bainov, S. I. Kostadinov, Nguyen Hong Thai and P. P. Zabreiko, "Existence of integral manifolds for impulsive differential equations", International Journal of Theoretical Physics, 28 (1989), No. 7, 815-833.

[8] M. Golubitsky and V. Guillemin, "Stable Mappings and Their Singularities", Spinger-Verlag, New York-Heideberg-Berlin, 1973.

[9] B. Aulbach, "Continuous and Discrete Dynamics near Manifolds of Equilibria, Lecture Notes in Mathematics", v. 1058, Springer-Verlag, Berlin-Heidelberg-New York-Tokyo, 1984.

10] D. D. Bainov, S. I. Kostadinov, P. P. Zabreiko and Nguyen Van Minh, "Topological equivalence and exponential dichotomy of linear impulsive equations", to appear.

[11] B. F. Bylov, R. E. Vinograd, D. M. Grobman and V. V. Nemytsky, Theory of Lyapunov Exponents, Nauka, Moscow, 1966 (in Russian).

[12] E. A. Tichonova, "Analogy and homeomorphism of perturbed and unperturbed systems with blocktriangular matrix",Differential Eqiations 7 (1970), 1221-1229 (in Russian).

Corresponding author : Drumi Bainov, P.O. Box 45, 1504 Sofia, Bulgaria. 